\title{
Gain spectroscopy of a type-II VECSEL chip
}

C. Lammers, M. Stein, C. Berger, C. Möller, C. Fuchs, A. Ruiz Perez, A. Rahimi-Iman, J. Hader, J. V. Moloney, W. Stolz, S. W. Koch, and M. Koch

Citation: Appl. Phys. Lett. 109, 232107 (2016); doi: 10.1063/1.4971333

View online: http://dx.doi.org/10.1063/1.4971333

View Table of Contents: http://aip.scitation.org/toc/apl/109/23

Published by the American Institute of Physics

\section{Articles you may be interested in}

III-Nitride-on-silicon microdisk lasers from the blue to the deep ultra-violet

Appl. Phys. Lett. 109, 231101231101 (2016); 10.1063/1.4971357

High power (60 mW) GaSb-based $1.9 \mu \mathrm{m}$ superluminescent diode with cavity suppression element Appl. Phys. Lett. 109, 231102231102 (2016); 10.1063/1.4971972

Interplay between quantum well width and interface roughness for electron transport mobility in GaAs quantum wells

Appl. Phys. Lett. 109, 232105232105 (2016); 10.1063/1.4971824

Terahertz master-oscillator power-amplifier quantum cascade lasers

Appl. Phys. Lett. 109, 231105231105 (2016); 10.1063/1.4969067

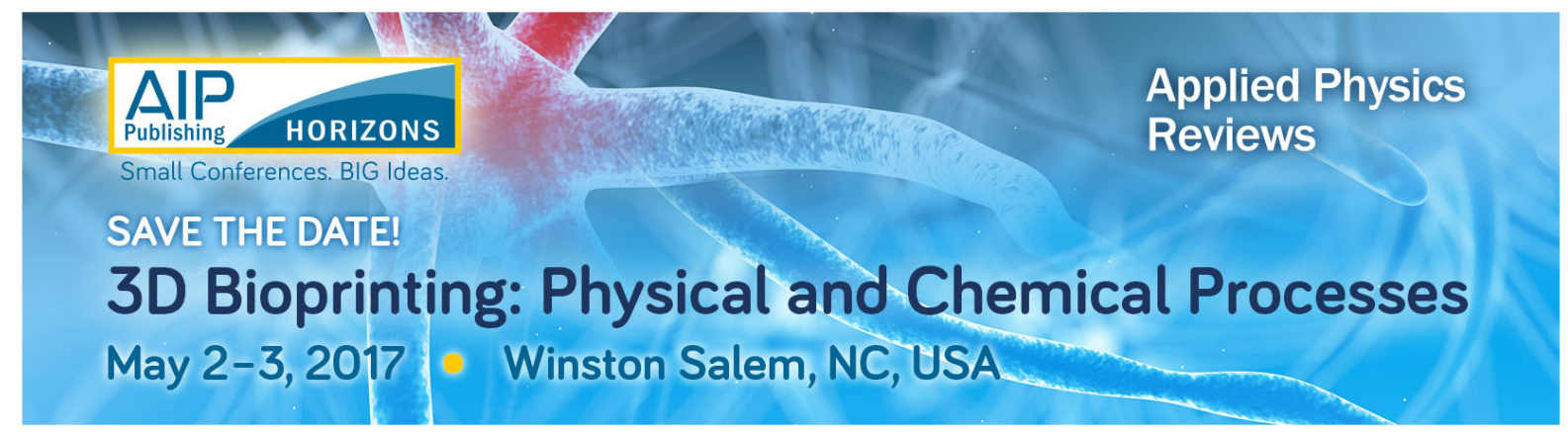




\title{
Gain spectroscopy of a type-II VECSEL chip
}

\author{
C. Lammers, ${ }^{1, a)}$ M. Stein, ${ }^{1}$ C. Berger, ${ }^{1}$ C. Möller, ${ }^{1}$ C. Fuchs, ${ }^{1}$ A. Ruiz Perez, ${ }^{2}$ \\ A. Rahimi-Iman, ${ }^{1}$ J. Hader, ${ }^{3}$ J. V. Moloney, ${ }^{3}$ W. Stolz, ${ }^{1}$ S. W. Koch, ${ }^{1}$ and M. Koch ${ }^{1}$ \\ ${ }^{1}$ Department of Physics and Material Sciences Center, Philipps-Universität Marburg, Renthof 5, \\ 35032 Marburg, Germany \\ ${ }^{2} N A s P_{I I / V}$ GmbH, Hans-Meerwein-Straße, 35032 Marburg, Germany \\ ${ }^{3}$ College of Optical Sciences, University of Arizona, 1630 E. University Blvd., Tucson, Arizona 85721, USA
}

(Received 19 August 2016; accepted 16 November 2016; published online 8 December 2016)

\begin{abstract}
Using optical pump-white light probe spectroscopy, the gain dynamics is investigated for a vertical-external-cavity surface-emitting laser chip, which is based on a type-II heterostructure. The active region of the chip consists of a GaAs/(GaIn)As/Ga(AsSb)/(GaIn)As/GaAs multiple quantum well. For this structure, a fully microscopic theory predicts a modal room temperature gain at a wavelength of $1170 \mathrm{~nm}$, which is confirmed by the experimental spectra. The results show a gain buildup on the type-II chip that is delayed relative to that of a type-I chip. This slower gain dynamics is attributed to a diminished cooling rate arising from the reduced electron-hole scattering. Published by AIP Publishing. [http://dx.doi.org/10.1063/1.4971333]
\end{abstract}

Very recently, laser operation has been demonstrated in a type-II vertical-external-cavity surface-emitting laser (VECSEL). ${ }^{1}$ The type-II design promises further wavelength flexibility in the infrared as well as reduced Auger losses. ${ }^{2,3}$ This makes lasers with a type-II based gain medium promising for many applications, such as optical data transmission. ${ }^{4}$

Important aspects of the optical properties and carrier dynamics of type-II quantum film structures have been investigated already 25 years ago. ${ }^{5-8}$ Recently, type-II semiconductor heterostructures with a "W"-type band alignment of the conduction band, spatially separating electron and hole confinement were found promising for laser applications, as reported in Refs. 9 and 10.

Up to date, both edge emitters and surface-emitting quantum well lasers based on type-II material systems have been demonstrated. ${ }^{11,12}$ The gain properties of such structures are often characterized by stationary methods such as the Hakki-Paoli technique or the variable-stripe-length method. ${ }^{13-16}$ Information on the gain dynamics associated with the carrier dynamics inside the laser medium can be obtained via ultrafast pump-probe experiments. ${ }^{17-19}$

Here, we use optical pump-white light probe spectroscopy to study the gain dynamics of a type-II "W"-VECSEL chip containing $10 \times(\mathrm{GaIn}) \mathrm{As} / \mathrm{Ga}(\mathrm{AsSb})$ "W"-multiple quantum wells. A fully microscopic theory predicts significant modal room temperature gain for this chip at a wavelength around $1170 \mathrm{~nm}$. For comparison, we additionally study a conventional type-I chip. We find that the gain build up is delayed in the type-II structure, as compared to the type-I chip. We attribute this slower dynamics to a reduced carrier cooling rate in the type-II structure.

Our VECSEL chip was grown bottom-up on exact GaAs (001) $\left( \pm 0.1^{\circ}\right)$ substrate in a horizontal AIXTRON AIX 200 Gas Foil Rotation (GFR) metal organic vapor phase epitaxy (MOVPE) reactor system. It consists of a lattice matched (GaIn)P capping layer, a resonant periodic gain (RPG) structure with 10 multi-quantum well heterostructures (MQWHs)

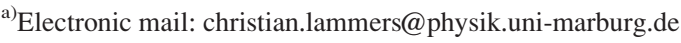

and a distributed Bragg reflector (DBR) consisting of 22 1/2 pairs of (AlGa)As/AlAs. The MQWH consists of a "W"shaped (GaIn)As/Ga(AsSb)/(GaIn)As type-II band alignment, which forms the active region, as can be seen in the inset of Fig. 1(b). The indirect transitions arise from electrons in the conduction band of the (GaIn)As quantum wells (QWs) and holes in the valence band of the $\mathrm{Ga}(\mathrm{AsSb}) \mathrm{QW}$ (dashed double arrows). At least three of them exist: the e1h1, e2h2, and e1h3 transitions, as can be seen in detail for a very similar structure in Ref. 10. The gain dynamics investigated here can be attributed to the elh1 transition. The active regions are separated by the $\mathrm{GaAs} / \mathrm{Ga}(\mathrm{AsP}) / \mathrm{GaAs}$ barriers. In order to obtain a resonant structure, the optical layer thicknesses of the barriers as well as the (GaIn)P capping layer were matched to $\lambda / 2$ with respect to the lasing wavelength. The layer thicknesses and compositions of the active region were determined by fitting a fully dynamical simulation to the high-resolution X-ray diffraction (HR-XRD) ((004)-reflection) pattern of the chip structure. This analysis yields a $\mathrm{Ga}\left(\mathrm{As}_{1-x} \mathrm{Sb}_{x}\right) \quad(\mathrm{x}=0.198)$

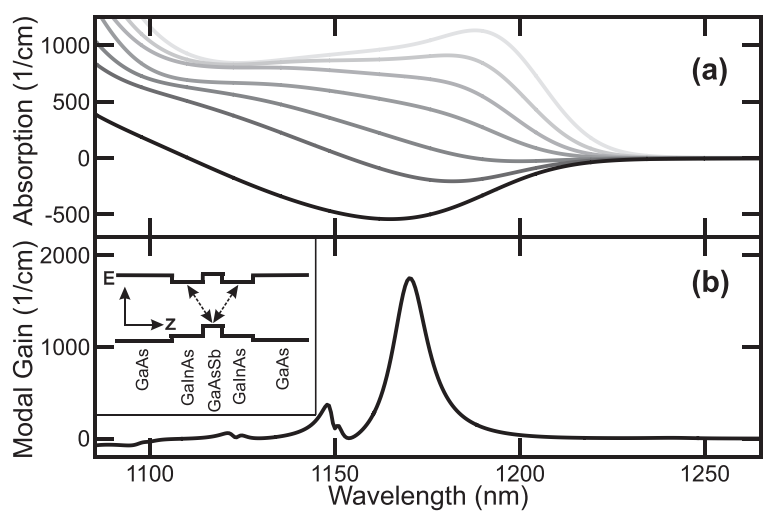

FIG. 1. Theoretical material absorption and modal gain. (a) Calculated material absorption for the active region of the type-II "W"-laser structure is presented for carrier densities ranging from $0.1 \times 10^{12} / \mathrm{cm}^{2}$ up to $3 \times 10^{12} /$ $\mathrm{cm}^{2}$ (bright to dark). (b) A simulation of the modal gain for the full type-II VECSEL system is plotted for a carrier density of $3 \times 10^{12} / \mathrm{cm}^{2}$. The inset schematically illustrates the band alignment of the type-II heterostructure. The dashed double arrows indicate the indirect radiative transitions. 
layer thickness of $4.0 \mathrm{~nm}$. The $\left(\mathrm{Ga}_{1-y} \mathrm{In}_{y}\right) \mathrm{As}(\mathrm{y}=0.203)$ layer has a thickness of $5.5 \mathrm{~nm}$.

In the following, we present calculations on the basis of the semiconductor Bloch equations (SBE) to predict the gain properties of this structure under optical excitation. In particular, we calculate the changes in absorption and refractive index for various carrier densities. ${ }^{20-22}$ To achieve a sufficient level of accuracy in our calculations, we include all terms in the SBE up to the second Born level to take homogeneous broadening into account, i.e., intrinsic microscopic carrier scattering inside the semiconductor. ${ }^{23,24}$ Unavoidable and common growth inhomogeneities in such MQWH samples are modeled by an inhomogeneous broadening, a convolution of our theoretical spectra with a Gaussian distribution describing the variation of the band gap energies. ${ }^{25}$ Starting point for our calculations are the band structure and the corresponding wavefunctions. All these single-particle properties are accessed evaluating an $8 \times 8$ multi band $\mathbf{k} \cdot \mathbf{p}$ model. ${ }^{23,26} \mathrm{We}$ assume all carriers to be in thermal equilibrium and therefore Fermi distributed in their respective bands. Due to the type-II design, local charge inhomogeneities can arise. Consequently, we solve the Schrödinger-Poisson equation to obtain the changes to the confinement potential. ${ }^{27}$ Based on the band structure and the single-particle wavefunctions, we compute the dipole and Coulomb matrix elements. ${ }^{24}$

Having modeled the absorption and refractive index changes induced by a given carrier density, we can derive the overall optical properties of the VECSEL structure. Reflection properties of the sample are calculated using the transfer-matrix method. ${ }^{22,28}$ From the transfer-matrix calculations, we obtain a spectral filter function that describes the spatial overlap of the quantum well positions inside the RPG with the intensity maxima of the longitudinal light modes of the optical cavity. ${ }^{29}$

The numerical results for the material absorption of the "W"-type MQWH at $300 \mathrm{~K}$ are presented in Fig. 1(a). Here, we assumed an inhomogeneous broadening of $20 \mathrm{meV}$. As the carrier density is increased from $0.1 \times 10^{12} / \mathrm{cm}^{2}$ up to $3 \times 10^{12} / \mathrm{cm}^{2}$ (bright to dark), the excitonic absorption peak vanishes and the absorption becomes negative. The calculations show that a spectrally broad gain region is formed for this material composition in the RPG. We compute the modal gain for a carrier density of $3 \times 10^{12} / \mathrm{cm}^{2}$, by multiplying the filter function (represented as dashed line in Fig. 2) with the gain of the RPG structure. Thereby, the broad material gain shown in Fig. 1(a) evolves into a narrow modal gain around $1170 \mathrm{~nm}$. This is shown in Fig. 1(b).

Next, we compare the simulated reflectivity to an experimental spectrum in Fig. 2. Here, the measured spectrum plotted in the background of the diagram represents the system under unexcited conditions (yellow shaded area). A direct comparison between experiment and theory shows a good qualitative agreement. Using our theory as a predictive tool, we increase the carrier density up to $3 \times 10^{12} / \mathrm{cm}^{2}$, which leads to the broad material gain, as observed in Fig. 1(a). Thereby, we observe a gradual increase in the reflectivity at the absorption dip in the stop band. Finally, the absorption dip evolves into a gain peak around $1170 \mathrm{~nm}$.

For our optical pump-white light probe gain measurements, we use a $1 \mathrm{kHz}, 5 \mathrm{~mJ}, 35 \mathrm{fs}$ regenerative Ti:sapphire

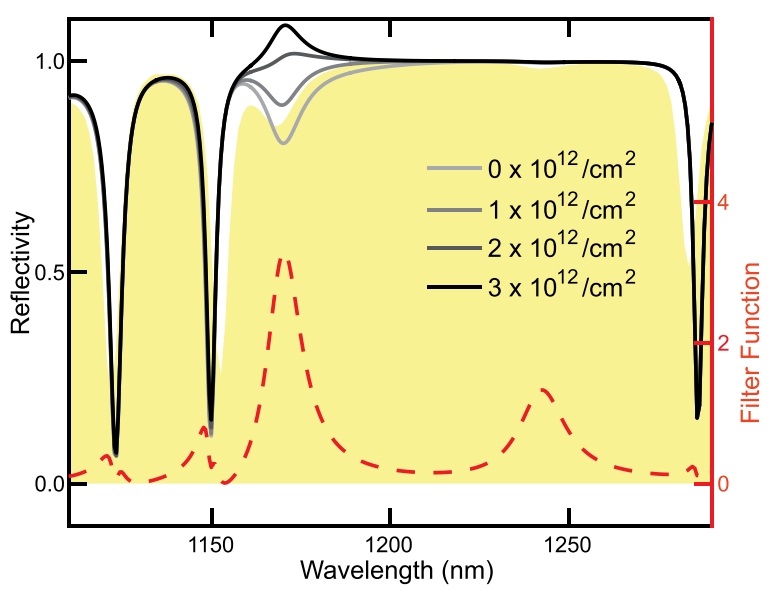

FIG. 2. Theoretical reflectivity (lines) of the type-II gain structure together with its calculated filter function (dashed line). Measured reflectance at room temperature for an unexcited chip is shown as yellow area in the background, while theoretical reflectivity spectra are presented for carrier densities between zero and $3 \times 10^{12} / \mathrm{cm}^{2}$ (solid lines, bright to dark). Again, the system temperature is set to $300 \mathrm{~K}$ with an inhomogeneous broadening of $20 \mathrm{meV}$.

amplifier to acquire time-resolved differential reflectivity spectra. The amplifier drives an optical parametric amplifier (OPA) to provide spectrally tunable fs pump pulses and furthermore generates an fs supercontinuum that is used to probe the gain dynamics in the chip. The pump spot diameter is determined to $150 \mu \mathrm{m}$ with a knife-edge measurement. An InGaAs-CCD cooled with liquid nitrogen is used as a detector in combination with a Czerny-Turner monochromator with an $80 \mathrm{l} / \mathrm{mm}$ diffraction grating as dispersive element. The excitation density of our pump pulse with a central wavelength of $1000 \mathrm{~nm}$ is set to $5.6 \times 10^{16} / \mathrm{cm}^{2}$ in order to inject a sufficiently large amount of charge carriers. All experiments are carried out at room temperature. To obtain the gain spectra, the measured differential reflectivity spectra were added to the reflectivity measurements of the unexcited sample.

Examples of the reflectivity spectra obtained for different times after the excitation pulse are shown in Fig. 3. For the unexcited sample, one clearly observes the dip in reflection at

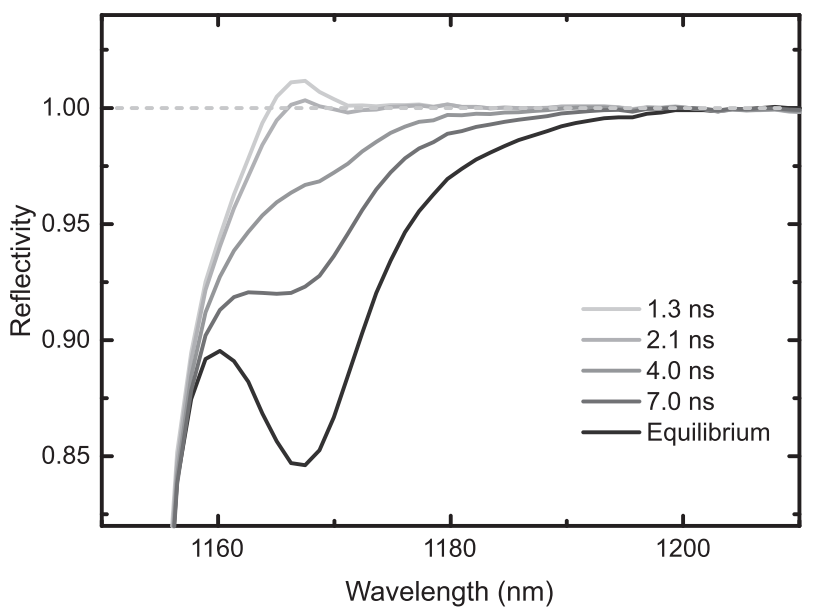

FIG. 3. Experimental reflectivity spectra of a type-II chip for pump-probe time delays between 1.3 and $7.0 \mathrm{~ns}$ (solid lines, bright to dark). The maximum gain is reached $1.3 \mathrm{~ns}$ after excitation. The equilibrium situation corresponds to the reflectivity measured by the probe pulse without excitation. 
$1168 \mathrm{~nm}$, which was predicted by theory. It is governed by an interplay of material absorption with the filter function for the employed material and structure, as indicated in Fig. 1. After excitation and carrier relaxation, the sample shows gain, i.e., the reflectivity exceeds one. The gain reaches its maximum after $1.3 \mathrm{~ns}$. It peaks at $1168 \mathrm{~nm}$ and has a spectral width of $6 \mathrm{~nm}$. The gain lasts for several hundred picoseconds. At 2.1 ns, it is barely noticeable. Thereafter, i.e., for larger delay times, the reflectivity in this spectral region is below one and gradually reduces.

The black curve in Fig. 4 shows the measured gain dynamics of the type-II chip. To obtain this curve, a region of $1.2 \mathrm{~nm}$ width around $1168 \mathrm{~nm}$ was spectrally integrated. The gain evolves after $600 \mathrm{ps}$, reaches its maximum after 1.3 ns and vanishes at $2.1 \mathrm{~ns}$ after the optical excitation. For comparison, the same measurements were performed on a type-I VECSEL, exploiting spatially direct transitions. The respective chip, which emits at $1101 \mathrm{~nm}$, has an RPG comprising $10\left(\mathrm{Ga}_{0.71} \mathrm{In}_{0.29}\right)$ As QWs with a thickness of $5 \mathrm{~nm}$ in between GaAs barriers. Again, the filter function is positioned at the edge of the stop band. Hence, all in all one has a similar arrangement as for the type-II VECSEL chip. The grey curve in Fig. 4 shows the gain dynamics of this chip. Once more, we spectrally integrate over a window of $1.2 \mathrm{~nm}$ around the gain maximum. In this case, the gain appears at $150 \mathrm{ps}$ and lasts for $1.1 \mathrm{~ns}$. By fitting exponential functions to the decreasing reflectivity, we retrieve charge-carrier lifetimes for both VECSEL structures. These lifetimes amount to $3.3 \mathrm{~ns}$ and $7.3 \mathrm{~ns}$ for the type-I and type-II chip, respectively. Although the type-I structure produces a higher peak value of 1.016, the time span of gain provided by the material system lasts longer for the type-II structure. As mentioned above, this can be seen as a direct effect of the spatial separation of charge carriers in type-II MQWHs, which exhibit a reduced recombination rate. Hence, high chargecarrier densities, which are a prerequisite for gain, are conserved for a longer period of time in comparison to a type-I heterostructure. It is reasonable to assume that a lower radiative recombination rate in a type-II structure can be a limiting factor with regard to the maximum amount of gain.

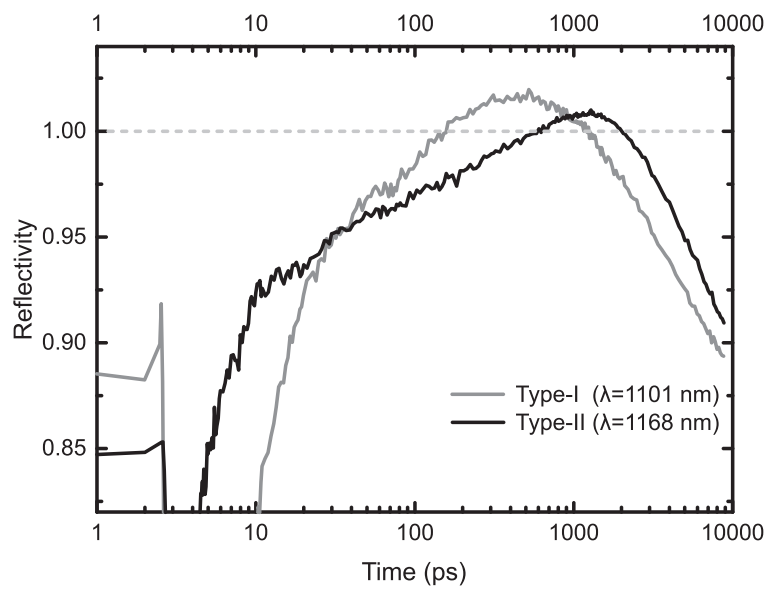

FIG. 4. Semilogarithmic plot showing the gain dynamics of the type-II "W"-VECSEL chip (black line) in comparison to a conventional type-I chip (grey line). For this plot, an offset of 2 ps was applied to the time axis in order to enable a logarithmic illustration.
Nonetheless, reducing the band gap energy in conventional type-I structures leads to an increase in non-radiative losses. Especially, by pushing the wavelength further to the telecom regime, type-II structures might overcome the performance of traditional type-I structures. In general, lowering band gap energies increases potential barrier heights, consequently reducing the overlap of electron and hole wavefunctions in a type-II alignment. However, for increasing carrier densities, band bending effects restore the overlap of the wavefunctions, thus maintaining or even increasing the gain. The slower gain build up in the type-II chip is attributed to a reduced carrier equilibration rate arising from the following situation: after optical excitation, depending on their excess energies, electron and hole distributions emit optical phonons and cool down. The cooling rates for electrons and holes are typically not identical and differ from material to material. Yet, in a type-I sample, electrons and holes can efficiently exchange energy via Coulomb scattering and, hence, the electron and hole distributions have a route to rapidly equilibrate among each other. If the electron distribution cools slower, it can profit from a hole distribution that cools faster-and vice versa. In a type-II sample, electron-hole scattering is significantly diminished due to the reduced spatial overlap of electrons and holes. Hence, it takes more time until a thermal equilibrium among the two distributions is achieved. Yet, a microscopic calculation of this effect for the multi-layer structure studied is beyond the scope of this paper.

In conclusion, we have investigated the gain dynamics of a type-II VECSEL chip using optical pump-white light probe spectroscopy. Experimental gain spectra agree well with a fully microscopic theory. We observe a slower buildup of the modal gain for the type-II chip when compared to a type-I structure. We attribute this to a smaller carrier cooling rate in the type-II chip arising from a reduced electron-hole scattering rate. The smaller scattering rate results from the reduced spatial overlap of electrons' and holes' wave functions in the type-II design. Owing to the fact that a type-II chip can be employed in VECSELs and that the design allows for more wavelength flexibility compared to type-I structures, further efforts will be done in the upcoming investigations in order to optimize the MQWH design with respect to gain and laser output. Recently, similar structures have been grown showing photoluminescence emission up to $1470 \mathrm{~nm} .^{30}$ Thereby, type-II VECSELs may push available emission wavelengths further to the highly desired telecom wavelengths.

The authors from Marburg acknowledge the financial support from the Deutsche Forschungsgemeinschaft via the Collaborative Research Center 1083 (DFG:SFB1083). The authors from Arizona are supported by the AFOSR Grant No. FA9550-14-1-0062.

${ }^{1}$ C. Möller, C. Fuchs, C. Berger, A. Ruiz Perez, M. Koch, J. Hader, J. V. Moloney, S. W. Koch, and W. Stolz, Appl. Phys. Lett. 108, 071102 (2016).

${ }^{2}$ W. W. Bewley, J. R. Lindle, C. S. Kim, M. Kim, C. L. Canedy, I. Vurgaftman, and J. R. Meyer, Appl. Phys. Lett. 93, 041118 (2008).

${ }^{3}$ J. R. Meyer, C. L. Felix, W. W. Bewley, I. Vurgaftman, E. H. Aifer, L. J. Olafsen, J. R. Lindle, C. A. Hoffman, M. J. Yang, B. R. Bennett, 
B. V. Shanabrook, H. Lee, C. H. Lin, S. S. Pei, and R. H. Miles, Appl. Phys. Lett. 73, 2857 (1998).

${ }^{4}$ E. Murphy, Nat. Photonics 4, 287 (2010).

${ }^{5}$ K. Meissner, B. Fluegel, R. Binder, S. W. Koch, G. Khitrova, and N. Peygambarian, Appl. Phys. Lett. 59, 259 (1991).

${ }^{6}$ R. Binder, I. Galbraith, and S. W. Koch, Phys. Rev. B 44, 3031 (1991).

${ }^{7}$ I. Galbraith, P. Dawson, and C. T. Foxon, Phys. Rev. B 45, 13499 (1992).

${ }^{8}$ J. Feldmann, R. Sattmann, E. O. Göbel, J. Kuhl, J. Hebling, K. Ploog, R. Muralidharan, P. Dawson, and C. T. Foxon, Phys. Rev. Lett. 62, 1892 (1989).

${ }^{9}$ C. Berger, C. Möller, P. Hens, C. Fuchs, W. Stolz, S. W. Koch, A. Ruiz Perez, J. Hader, and J. V. Moloney, AIP Adv. 5, 047105 (2015).

${ }^{10}$ S. Gies, C. Kruska, C. Berger, P. Hens, C. Fuchs, A. Ruiz Perez, N. W. Rosemann, J. Veletas, S. Chatterjee, W. Stolz, S. W. Koch, J. Hader, J. V. Moloney, and W. Heimbrodt, Appl. Phys. Lett. 107, 182104 (2015).

${ }^{11}$ J. F. Klem, O. Blum, S. R. Kurtz, I. J. Fritz, and K. D. Choquette, J. Vac. Sci. Technol. 18, 1605 (2000).

${ }^{12}$ J. Meyer, L. Olafsen, E. Aifer, W. Bewley, C. Felix, V. Vurgaftman, M. Yang, L. Goldberg, D. Zhang, C.-H. Lin, S. Pei, and D. Chow, IEE Proc.-Optoelectron. 145, 275 (1998).

${ }^{13}$ B. W. Hakki, J. Appl. Phys. 44, 4113 (1973).

${ }^{14}$ L. Shterengas, R. Liang, G. Kipshidze, T. Hosoda, S. Suchalkin, and G. Belenky, Appl. Phys. Lett. 103, 121108 (2013).

${ }^{15}$ K. L. Shaklee, R. E. Nahory, and R. F. Leheny, JOL 7, 284 (1973).

${ }^{16}$ N. Kirstaedter, O. G. Schmidt, N. N. Ledentsov, D. Bimberg, V. M. Ustinov, A. Y. Egorov, A. E. Zhukov, M. V. Maximov, P. S. Kop'ev, and Z. I. Alferov, Appl. Phys. Lett. 69, 1226 (1996).

${ }^{17}$ K. L. Hall, J. Mark, E. P. Ippen, and G. Eisenstein, Appl. Phys. Lett. 56, 1740 (1990).
${ }^{18}$ H. Giessen, U. Woggon, B. Fluegel, G. Mohs, Y. Z. Hu, S. W. Koch, and N. Peyghambarian, Opt. Lett. 21, 1043 (1996).

${ }^{19}$ C. Lange, S. Chatterjee, C. Schlichenmaier, A. Thränhardt, S. W. Koch, W. W. Rühle, J. Hader, J. V. Moloney, G. Khitrova, and H. M. Gibbs, Appl. Phys. Lett. 90, 251102 (2007).

${ }^{20}$ M. Lindberg and S. W. Koch, Phys. Rev. B 38, 3342 (1988).

${ }^{21}$ H. Haug and S. W. Koch, Quantum Theory of the Optical and Electronic Properties of Semiconductors, 5th ed. (World Scientific Publication, Singapore, 2009).

${ }^{22}$ M. Kira and S. W. Koch, Semiconductor Quantum Optics (Cambridge University Press, Cambridge, 2012).

${ }^{23}$ W. W. Chow and S. W. Koch, Semiconductor-Laser Fundamentals: Physics of the Gain Materials (Springer, Berlin, Heidelberg/New York, 1999).

${ }^{24}$ J. Hader, S. W. Koch, and J. V. Moloney, Solid-State Electron. 47, 513 (2003).

${ }^{25}$ J. Hader, J. V. Moloney, S. W. Koch, and W. W. Chow, IEEE J. Sel. Top. Quantum Electron. 9, 688 (2003).

${ }^{26}$ J. Hader, N. Linder, and G. H. Döhler, Phys. Rev. B 55, 6960 (1997).

${ }^{27}$ D. Ahn and S. L. Chuang, J. Appl. Phys. 64, 6143 (1988).

${ }^{28} \mathrm{M}$. Born and E. Wolf, Principles of Optics, 7 th ed., Electromagnetic Theory of Propagation, Interference and Diffraction of Light (Cambridge University Press, Cambridge, 1999).

${ }^{29}$ M. Schafer, W. Hoyer, M. Kira, S. W. Koch, and J. V. Moloney, J. Opt. Soc. Am. B 25, 187 (2008).

${ }^{30}$ C. Fuchs, A. Beyer, K. Volz, and W. Stolz, "MOVPE growth of (GaIn)As/ $\mathrm{Ga}(\mathrm{AsSb}) /(\mathrm{GaIn}) \mathrm{As}$ type-II heterostructures on GaAs substrate for near infrared laser applications" J. Cryst. Growth (in press). 